\title{
Astragaloside IV protects retinal pigment epithelial cells from apoptosis by upregulating miR-128 expression in diabetic rats
}

\author{
TAO WANG ${ }^{*}$, ZHONGWEI ZHANG ${ }^{*}$, CAIPING SONG, LEI SUN, XINLI SUI, QUN QU and JING LIU \\ Department of Ophthalmology, Weihai Municipal Hospital, Weihai, Shandong 264200, P.R. China
}

Received January 13, 2020; Accepted March 30, 2020

DOI: $10.3892 /$ ijmm.2020.4588

\begin{abstract}
The present study aimed to investigate the protective effects exerted by astragaloside-IV (AIV) on retinal pigment epithelial (RPE) cells of rats with diabetes mellitus (DM), and to explore the underlying molecular mechanisms. For this purpose, a rat model of DM was established by injecting rats with an intraperitoneal injection of streptozotocin. AIV was then intragastrically administered. An electroretinogram (ERG) was used to assess retinopathy and TUNEL staining was used to detect the level of apoptosis of RPE cells. Western blot analysis was used to determine protein expression in RPE cells in vitro and in vivo. AIV was found to be able to significantly increase body weight and decrease blood glucose levels in rats with DM in a dose-dependent manner. Compared with the rats with DM, the rat rod cell response a wave, $b$ wave, maximum response $b$ wave, photopic (photo)-ERG b wave and oscillatory potential (OP) p4 wave latency significantly decreased and the amplitude of $\mathrm{OP}$ Os1 wave increased significantly in the rats with DM treated with AIV for 11 weeks. In addition, AIV significantly decreased the apoptotic levels of RPE cells from rats with DM and significantly decreased the protein expression levels of Bax/Bcl-2, Fas/FasL, active caspase-3, active caspase-8, active caspase-9, homeobox B3 (HOXB3), p-phosphoinositide 3-kinase (PI3K)/PI3K, p-AKT/AKT and p-p70S6K1/p70S6K1, whereas it significantly increased miR-128 expression in the RPE cells from rats with DM. In vitro, AIV significantly inhibited the high glucose (HG)-induced apoptosis of RPE cells by increasing miR-128 expression and Bcl-2 and FasL protein expression in vivo. On the whole, the findings of the present study demonstrate that AIV treatment protects RPE cells of diabetic rats from apoptosis, and that these effects may be associated with the upregulation of miR-128 expression.
\end{abstract}

Correspondence to: $\mathrm{Dr}$ Caiping Song, Department of Ophthalmology, Weihai Municipal Hospital, 70 Heping Road, Huancui, Weihai, Shandong 264200, P.R. China

E-mail: songcaiping2020@sina.com

${ }^{*}$ Contributed equally

Key words: Astragalus polysaccharide, retinal pigment epithelial cells, miR-128, diabetes

\section{Introduction}

Diabetic retinopathy (DR) is a disease secondary to impaired glucose tolerance and retinal damage, which causes decreased vision that can even lead to macular edema, retinal detachment and vitreous hemorrhage, eventually resulting in blindness, and is one of the most common complications of diabetes mellitus (DM) (1). Data from the World Health Organization indicate that DR has become a leading cause of blindness among the working-age population in the United States (2), and is the 5th leading cause of blindness worldwide, while the number of patients with DR worldwide have been estimated to increase to 191 million by the year 2030 (3). Previous studies have found that sustained high glucose (HG) levels cause damage to retinal pigment epithelial (RPE) cells in diabetic patients, resulting in structural and secretory dysfunction, which proves that RPE cell damage plays an important role in the early development of DR (4).

RPE cells are derived from embryonic optic vesicles and play a vital role in the growth, development and visual function of the eye, and have also been found to exert antioxidant functions, maintain secretory growth factors and participate in physiological functions, such as circulating metabolism $(5,6)$. Moreover, RPE cells have been found to exhibit high levels of apoptosis in in vitro models, resulting from causes, such as oxidative stress (7) and blue light damage (8). Therefore, the apoptosis of RPE cells plays an important role in the pathogenesis of retinal degenerative diseases; the protection of RPE cells and the effective control of the apoptosis of RPE cells may delay the development of retinal degeneration (9).

Astragaloside-IV (AIV) is one of the main active ingredients extracted from Astragalus, and has been proven to exert anti-inflammatory, antioxidant, energy metabolism, neuroprotection and anticancer effects. AIV has also been used in the treatment of cardiovascular and cerebrovascular diseases, kidney diseases, respiratory diseases, diabetes and diabetes-related complications $(10,11)$. In addition, a previous study by the authors found that miR-128 attenuates the apoptosis of RPE cells induced by HG levels in vitro (12). miR-128 has also been found to be associated with insulin resistance $(13,14)$ and neuropathic susceptibility $(15)$ in diabetic patients. Although AIV has been found to exert protective effects on diabetic mouse retinopathy (16), its specific molecular mechanisms of action remain unclear, and it is unknown whether it is related to the inhibition of RPE cell apoptosis 
and if its effects involve miR-128. In the present study, the role and mechanisms of action of AIV in DR in rats with DM were investigated. A rat model of DM was established by an intraperitoneal injection of streptozotocin (STZ). It was found that AIV protected RPE cells from rats with DM from apoptosis by upregulating miR-128 expression, which in turn attenuated retinopathy in rats with DM.

\section{Materials and methods}

Experimental animal and grouping. The animal experiments performed in the present study were approved and supervised by the Animal Care and Use Committee of Weihai Municipal Hospital, and conformed with guidelines of the National Institution of Health. A total of 38 Sprague-Dawley (SD) rats (SPF) were used in the present study, and were kept under the following conditions: Temperature, $20-24^{\circ} \mathrm{C}$, humidity, $50-65 \%$ humidity, free access to food and water and 12-h light/dark cycle. A rat model of DM was established by injecting rats with an intraperitoneal injection of $100 \mathrm{mg} / \mathrm{kg}$ STZ (V900890; Sigma-Aldrich; Merck KGaA) using SD rats (male:female ratio, 1:1; 6 weeks old; weighing 180-200 g). The day of the injection of STZ in rats was defined as the first day. Blood glucose levels from the tail vein were measured on the third day after the STZ injection. Rats with a tail vein blood glucose level of $>16.7 \mathrm{mmol} / \mathrm{l}$ were defined as rats with DM. The rats in the Sham group were intraperitoneally injected with the same dose of a solvent (stroke-physiological saline solution) on the first day. The rats with DM were randomly divided into 3 groups, namely the DM group, the DM + L-AIV group, the DM + M-AIV group and the DM + H-AIV group, where $\mathrm{L}, \mathrm{M}$ and $\mathrm{H}$ represent low, medium, and high doses of AIV, respectively. The rats in the DM + L-AIV, DM + M-AIV and DM + H-AIV groups were intragastrically administered 20, 40 and $60 \mathrm{mg} / \mathrm{kg}$ AIV (Xi'an Sobeo Pharmaceutical Technology Co., Ltd.) daily, respectively. The other rats were administered the same volume of solvent. Rat weight and blood glucose levels were measured at the $0,4,8$ and 12th week of AIV administration and retinal function was evaluated using an electroretinogram (ERG) at the 12th week.

RPE cell separation and experiments. Rats were anesthetized by intraperitoneal injection of sodium pentobarbital at $80 \mathrm{mg} / \mathrm{kg}$, and the anesthesia status of the rats was determined by observing righting reflex, eye reflex, swallow reflex, pedal reflex and tail reflex. Subsequently, the deeply anesthetized rats were sacrificed by cervical dislocation, and the death of the rats was confirmed by observing breathing and heartbeat. After the SD rats were euthanized, their eyeballs were removed under aseptic conditions. The eyeballs were immersed in physiological saline containing $400 \mathrm{U} / \mathrm{ml}$ gentamicin (E003632; Sigma-Aldrich; Merck KGaA) for 30 min on ice. The eyeballs were then placed in $50 \mathrm{U} / \mathrm{ml}$ hyaluronidase (H1115000; Sigma-Aldrich; Merck KGaA) and $105 \mathrm{U} / \mathrm{ml}$ collagenase (1148089; Sigma-Aldrich; Merck KGaA) mixture buffered at $37^{\circ} \mathrm{C}$ for $45 \mathrm{~min}$. After washing 3 times with D-Hanks buffer, the eyeball was placed in $0.1 \%$ Trypsin (15090046, Thermo Fisher Scientific, Inc.) at $37^{\circ} \mathrm{C}$ for $30 \mathrm{~min}$. The eyeballs were dissected under an optical microscope (CX23, Olympus Corporation) to harvest RPE-choroid-sclera tissue, and were digested using $0.25 \%$ trypsin for $5 \mathrm{~min}$ at room temperature. Finally, the cell suspension was harvested by washing the tissue using the medium. The cell suspensions were incubated with $0.25 \%$ trypsin for $5 \mathrm{~min}$ at room temperature after removing the medium through centrifugation $(500 \mathrm{x} \mathrm{g}$, room temperature, $5 \mathrm{~min}$ ). A portion of the RPE cells were used directly to extract RNA for reverse transcription-quantitative PCR (RT-qPCR) analysis and protein for western blot analysis, while the other RPE cells were cultured at $37^{\circ} \mathrm{C}$ with $5 \% \mathrm{CO}_{2}$ in DMEM (12491-15, Thermo Fisher Scientific, Inc.) supplemented with $10 \%$ fetal bovine serum (10100-147, Thermo Fisher Scientific, Inc.). As described in a previous study by the authors (12), $50 \mu \mathrm{mol}$ miR-128-inhibitor (5'-AGUGUCACU UGGCCAGAGAAA-3'; Sangon Biotech) was first transfected into the RPE cells using Lipofectamine ${ }^{\mathrm{TM}} 2000$ transfection reagent (11668019, Invitrogen; Thermo Fisher Scientific, Inc.). After $72 \mathrm{~h}$, the cells were transferred to $4.5 \mathrm{~g} / \mathrm{l} \mathrm{HG}$ medium. The normal cell medium contained $1.0 \mathrm{~g} / 1$ glucose.

MTT assay. A total of $2 \times 10^{3}$ cells were seeded into a 96-well cell culture plate and cultured under normal conditions for $72 \mathrm{~h}$. The cell culture medium was then removed and $4.5 \mathrm{~g} / \mathrm{l}$ HG medium was added for 6, 12, 24 or $48 \mathrm{~h}$. Cell viability was then measured using a Cell Proliferation Assay kit (C0009, Beyotime Institute of Biotechnology).

TUNEL staining. The rat retinal tissues were fixed using paraformaldehyde and were made into paraffin-embedded sections, and TUNEL staining was used to detect the apoptosis of RPE cells in each group. All reagents and procedures used for TUNEL assay were according to the instructions provided by the manufacturer of the TUNEL Cell Apoptosis Detection kit (TA201-02, TransGen Biotech Co., Ltd.).

Western blot analysis. RIPA lyssis buffer (R0010, Solarbio, Inc.) was used to extract total protein from the RPE cells of the rats and the protein concentration was detected using a BCA kit (P0011, Beyotime Institute of Biotechnology), and $40 \mu \mathrm{g}$ of total protein were separated using 10\% SDS-PAGE under a constant voltage of $90 \mathrm{~V}$. The proteins were then transferred from the SDS-PAGE gel onto a PVDF membrane. After the samples were blocked using 5\% skimmed milk at room temperature for $1 \mathrm{~h}$, the membrane was incubated with the primary antibodies overnight at $4{ }^{\circ} \mathrm{C}$. The secondary antibody was used for incubation at room temperature for $2 \mathrm{~h}$. After being washed 3 times using phosphate-buffered saline/Tween-20, ECL solution (WBKLS0100, Beijing Xinjingke Biotechnologies Co., Ltd.) was added for detection, followed by densitometric analysis using Image J 3.0 software (IBM, Inc.) and $\beta$-actin was used as a loading control. Information pertaining to the antibodies used in the present study is presented in Table I.

$R T$ - $q P C R$. An RNA extract kit (9767, Takara, Inc.) was used to extract total RNA from the RPE cells of rats, and cDNA was constructed using a one-step cDNA reverse transcription kit (D0401, HaiGene). Finally, $20 \mu \mathrm{l}$ of the qPCR reaction solution were configured according to the instructions of the GoTaq qPCR Master Mix (A6006A, Promega Corp.), and the relative expression level of miR-128 against U6 RNA (internal control) was calculated using the $2^{-\Delta \Delta \mathrm{Cq}}$ method (17). The 
Table I. Antibody information.

\begin{tabular}{lccc}
\hline Antibody & Dilution & Cat. no. & Manufacturer \\
\hline Bcl-2 & $1: 2,000$ & 15071 & Cell Signaling Technology, Inc. \\
Bax & $1: 2,000$ & 5023 & Cell Signaling Technology, Inc. \\
Fas & $1: 1,000$ & 4233 & Cell Signaling Technology, Inc. \\
FasL & $1: 1,500$ & 68405 & Cell Signaling Technology, Inc. \\
Active caspase-3 & $1: 500$ & 9661 & Cell Signaling Technology, Inc. \\
Active caspase-8 & $1: 1,000$ & 9748 & Cell Signaling Technology, Inc. \\
Active caspase-9 & $1: 1,000$ & 9505 & Cell Signaling Technology, Inc. \\
HOXB3 & $1: 2,000$ & ab83404 & Abcam \\
PI3K & $1: 1,500$ & ab180967 & Abcam \\
p-PI3K & $1: 1,000$ & 17366 & Cell Signaling Technology, Inc. \\
AKT & $1: 2,000$ & ab18785 & Abcam \\
p-AKT & $1: 1,000$ & ab38449 & Abcam \\
p70s6k1 & $1: 1,500$ & ab32359 & Abcam \\
p-p70s6k1 & $1: 500$ & ab59208 & Abcam \\
$\beta$-actin & $1: 5,000$ & HC201 & TransGen Biotech Co., Ltd. \\
Goat anti-rabbit & $1: 3,000$ & ab6721 & Abcam \\
Goat anti-mouse & $1: 3,000$ & ab205719 & Abcam \\
\hline
\end{tabular}

following PCR primers were used: miR-128-F, 5'-CAAAGA CTACTGTGTAACTGCGA-3' and miR-128-R, 5'-TGGACT GTACTTGACAATGTTGG-3'; $\beta$-actin-F, 5'-GGCTGTATT CCCCTCCATCG-3'; U6-F, 5'-CTCGCTTCGGCAGCACA-3' and U6-R, 5'-AACGCTTCACGAATTTGCGT-3'.

Flow cytometric analysis for apoptosis. RPE cells subjected to the different treatments were harvested through centrifugation (500 x g, $5 \mathrm{~min}$, room temperature), and were washed 2 times with pre-cooled PBS on ice. An Annexin V-FITC/PI Apoptosis Detection kit was used to stain the cells and apoptotic cells were analyzed using a flow cytometer (Flow-Count, Beckman Coulter, Inc.). All procedures were performed according to the instructions of the manufacturers, and the measurements were repeated 2 times for each sample.

Statistical analysis. GraphPad prism v8.3.0 sofware was used to record and analyze the data collected. Data are expressed as the means \pm SD of 3 separate experiments, and one-way ANOVA with Tukey's test as a post hoc test was used to compare differences between multiple groups. A P-value $<0.05$ was considered to indicate a statistically significant difference.

\section{Results}

AIV attenuates retinal damage in diabetic rats. Through the intragastric administration of AIV daily, 1 week after the intraperitoneal injection of STZ, the body weight of the rats and blood glucose levels were measured at the 4,8 and 12th week. As shown in Fig. 1A, the body weight of the normal rats in the Sham group increased during the study period; however, the body weight of the rats with DM decreased in the 4th week and then slowly increased, while the body weight of the rats with DM treated with AIV was significantly higher than that of the rats with DM. Moreover, the body weight of the rats with
DM treated with a high dose of AIV was higher than those administered a medium dose of AIV, while the body weight of the rats with DM administered a low dose of AIV treatment was the lowest. It was also found that (Fig. 1B) the blood glucose levels of rats with DM were maintained at a high level; however, AIV treatment decrease the blood glucose levels of rats with DM in a dose-dependent manner, as the body weight of the rats changed.

Furthermore, retinal function was evaluated using an ERG, and it was found that (Fig. 1C) compared with the rats in the Sham group, the rat rod cell response a wave, b wave, maximum response $b$ wave, photopic-ERG $b$ wave and oscillatory potential (OP) p4 wave latency increased significantly, and the amplitude of OP os1 wave decreased significantly in rats with DM. However, following 11 weeks of $60 \mathrm{mg} / \mathrm{kg}$ AIV (H-AIV) treatment, the rat rod cell response a wave, b wave, maximum response $\mathrm{b}$ wave, phot-ERG $\mathrm{b}$ wave, OP $\mathrm{p} 4$ wave latency decreased significantly and the amplitude of OP Os1 wave increased significantly in DM rats.

AIV attenuates the apoptosis of REP cells from diabetic rats. At the 12th week, the rats were euthanized to obtain retinal tissues and RPE cells isolated. First, TUNEL staining was performed to measure the level of apoptosis of RPE cells from DM rats. It was found that (Fig. 2A) RPE cells from normal rats did not undergo apoptosis; however, the apoptosis of RPE cells from rats with DM was detected and the proportion of apoptotic RPE cells decreased significantly following treatment with AIV. Subsequently, apoptosis-related protein expression was detected by western blot analysis. It was and found that (Fig. 2B and C) the protein expression levels of Bcl-2, Bcl-2/Bax and FasL decreased significantly in the RPE cells from rats with DM, whereas AIV treatment significantly increased these expression levels. The protein expression levels of Bax, Fas, Fas/FasL, active caspase-3, active caspase- 8 and 

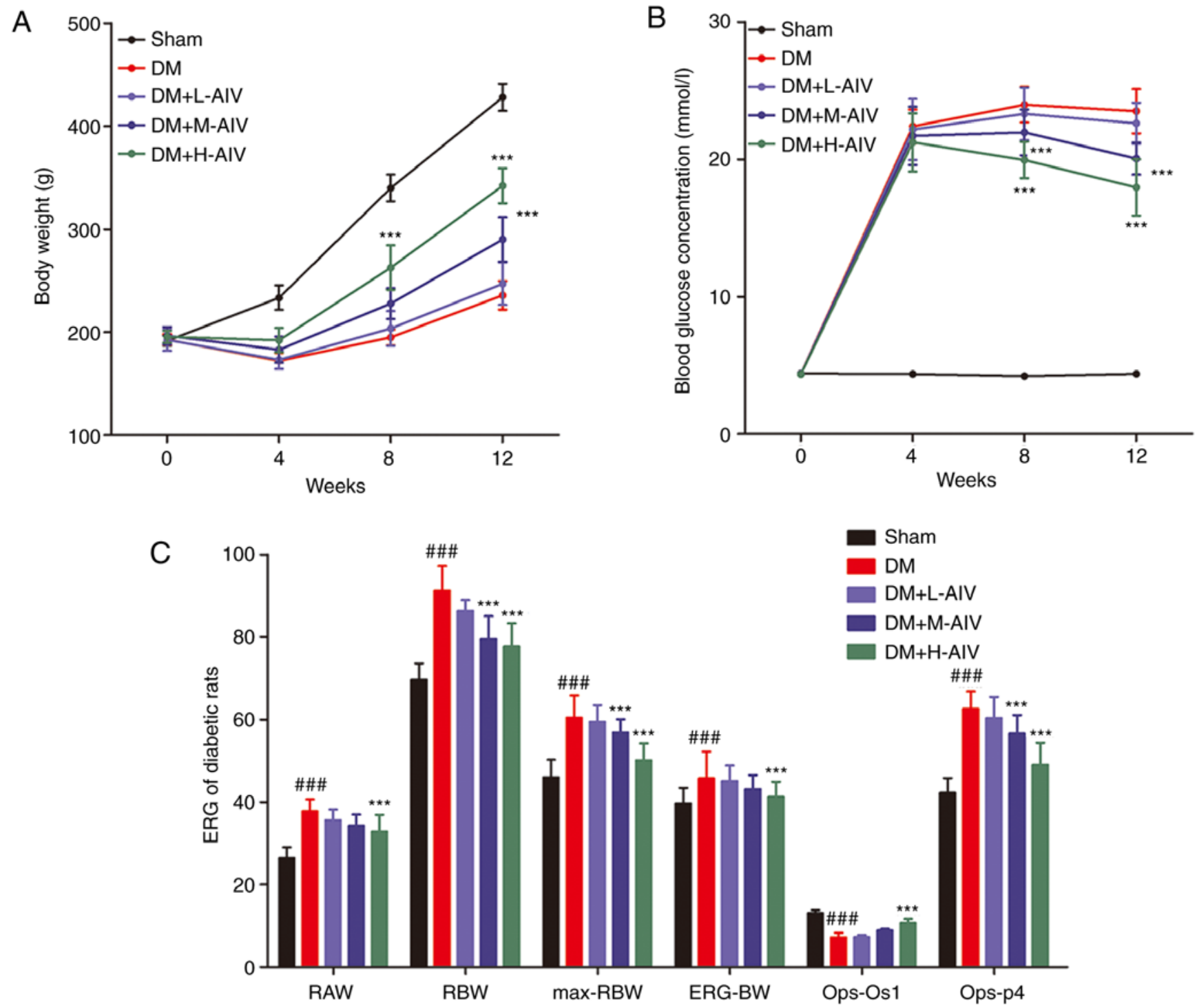

Figure 1. Effect of AIV on the ERG of DM rats. (A) AIV significantly increased the body weight of rats with DM in a dose-dependent manner; (B) AIV significantly induced blood glucose levels of rats with DM in a dose-dependent manner; (C) AIV significantly promoted the ERG of rats with DM at 12 weeks; 6 rats were in the Sham group, and 8 rats were in the other groups; ${ }^{* * *} \mathrm{P}<0.001 \mathrm{vs}$. DM group, and ${ }^{\# \# \#} \mathrm{P}<0.001$ vs. Sham group. Data are expressed as the means $\pm \mathrm{SD}$ of 3 individual experiments. AIV, astragaloside-IV; DM, diabetes mellitus; ERG, electroretinogram; RAW, rod cell response a wave; RBW, rod cell b wave; max-RBW, maximum response $b$ wave; ERG-BW, phot-ERG $b$ wave; Ops, oscillatory potentials.

active caspase-9 significantly increased in the RPE cells from rats with DM; however, AIV treatment significantly decreased these expression levels.

AIV increases miR-128 expression in REP cells from diabetic rats. A previous study by the authors demonstrated that miR-128 protects human RPE cells (ARPE-19) under HG conditions through the homeobox B3 (HOXB3)/phosphoinositide 3-kinase (PI3K)/extracellular regulated kinase (ERK)/mammalian target of rapamycin (mTOR) pathway (12). Therefore, the present study also detected the expression of miR-128 in RPE cells of rats. It was found that (Fig. 3A) miR-128 expression in RPE cells of DM rats was significantly lower than that in RPE cells from normal rats; however, miR-128 expression in RPE cells from DM rats treated with AIV (60 mg/kg) for 11 weeks increased significantly, compared with that in RPE cells from rats with DM. In addition, the expression of HOXB3, a target gene of miR-128, and the PI3K/AKT pathway was detected in RPE cells from the rats. As shown in Fig. 3B-F, the protein expression levels of HOXB3, p-PI3K/PI3K, p-AKT/AKT and p-p70S6K1/p70S6K1 in RPE cells from rats with without treatment were significantly higher than those in RPE cells from normal rats. However, after 11 weeks of AIV treatment, the elevated protein expression levels of HOXB3, p-PI3K/PI3K, p-AKT/AKT and p-p70S6K1/p70S6K1 decreased significantly.

AIV reduces the HG-induced apoptosis of REP cells in vitro. RPE cells were isolated from normal rats and treated with $\mathrm{HG}$, AIV or an miR-128-inhibitor. First, it was found that the viability of RPE cells gradually decreased along with the prolongation of HG treatment (Fig. 4A). The time point of $24 \mathrm{~h}$ was selected as the duration for RPE cells to be exposed to HG levels in the following experiments. Second, as shown in Fig. 4B, compared with the control group, the expression of miR-128 significantly decreased following exposure to HG; however, AIV significantly increased its expression. Subsequently, flow cytometry 

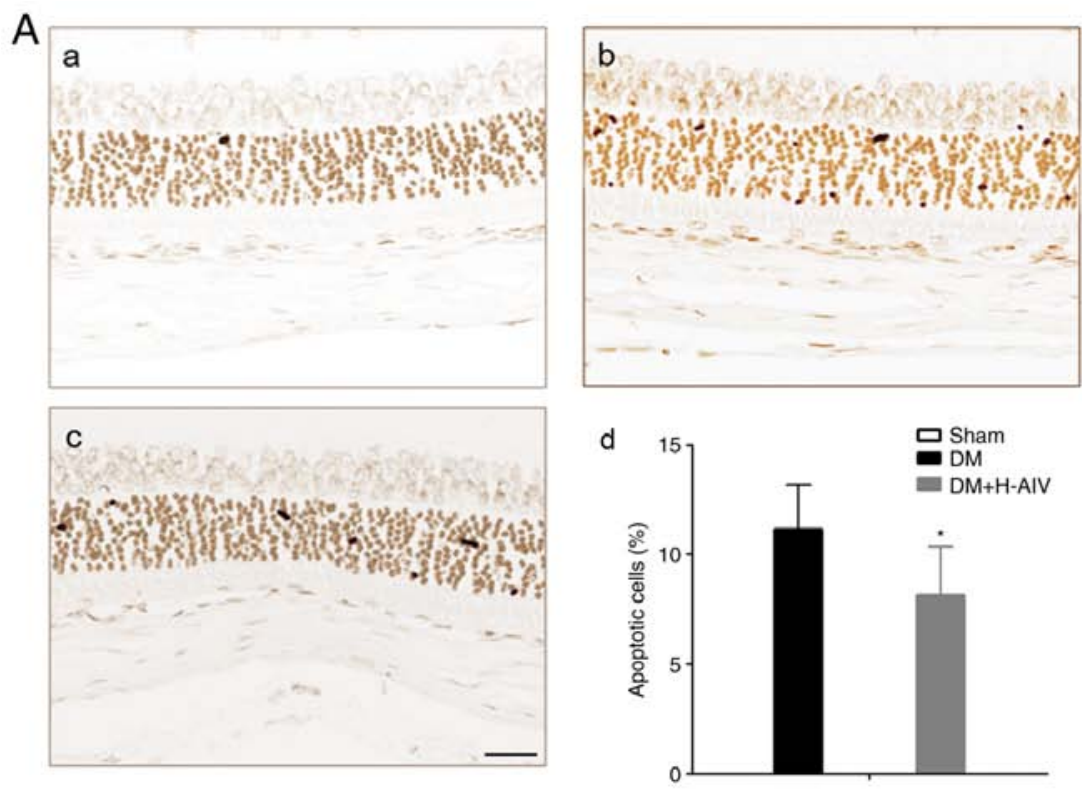

B

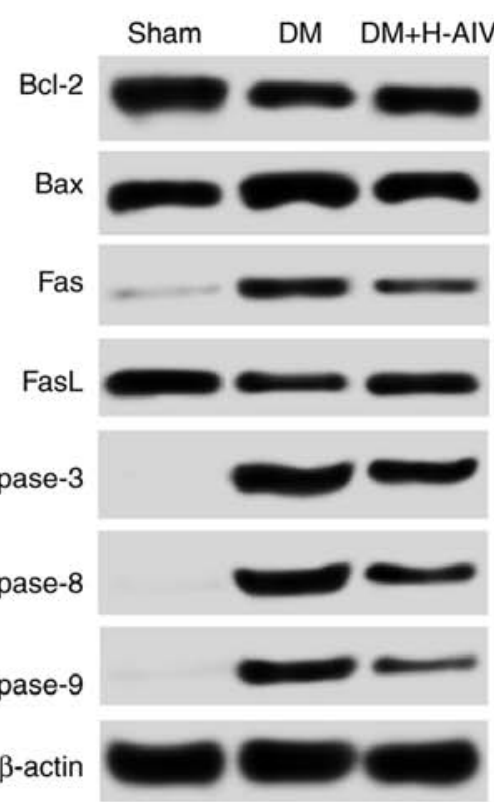

C
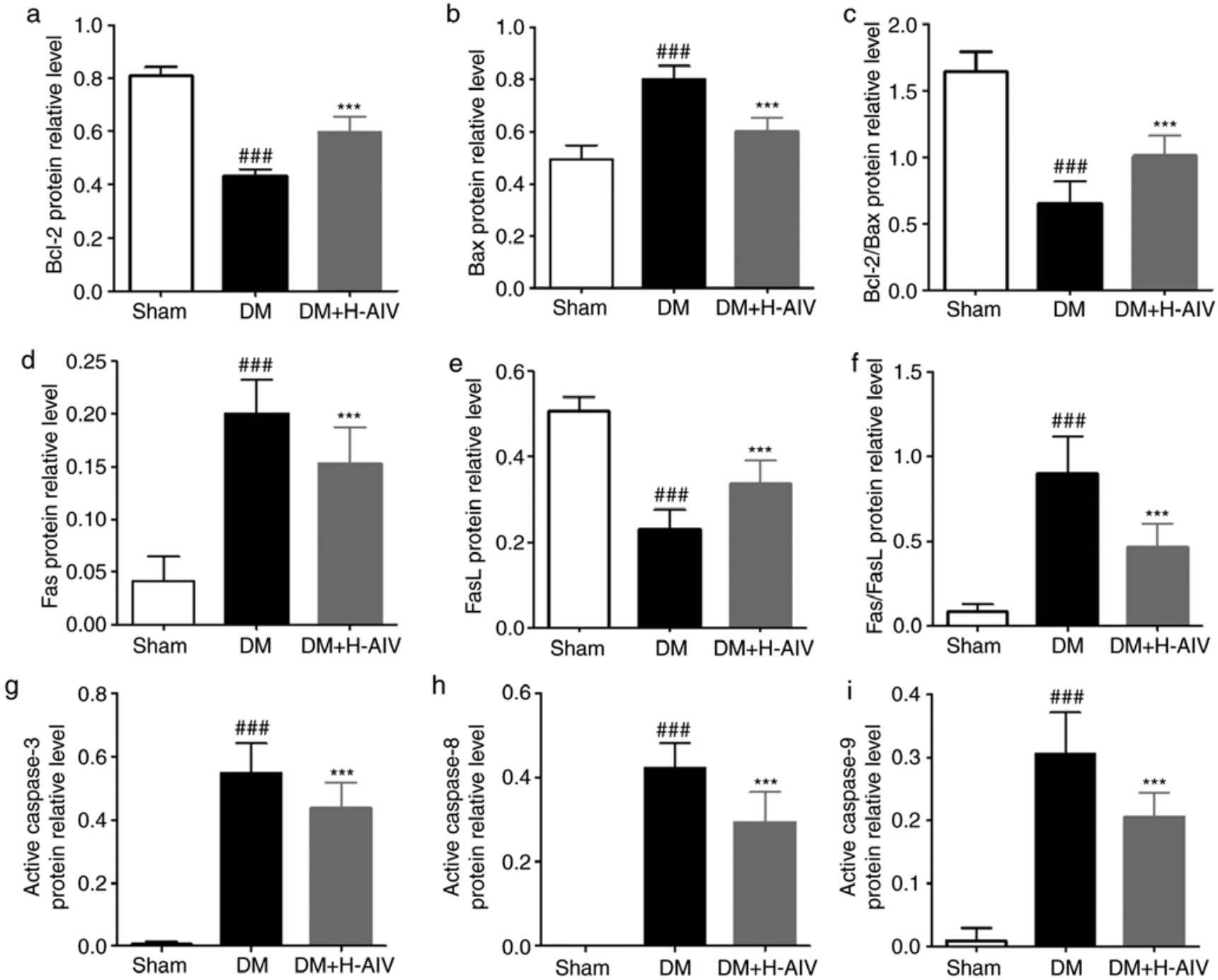

Figure 2. Effect of AIV on the apoptosis of RPE cells from rats with DM. (A) AIV significantly decreased the apoptosis of RPE cells from rats with DM as shown by TUNEL assay, (a) Sham, (b) DM, (c) DM+H-AIV, (d) percentage apoptotic cells; scale bar, $100 \mu \mathrm{m}$; (B) representative apoptosis-related protein expression bands in RPE cells from rats with DM; (C) statistical analysis of gray value of apoptosis-related protein bands, (a) Bcl-2, (b) Bax, (c) Bcl-2/Bax, (d) Fas, (e) FasL, (f) Fas/FasL, (g) active caspase-3, (h) active caspase-8, (i) active caspase-9. ${ }^{*} \mathrm{P}<0.05$ and ${ }^{* * *} \mathrm{P}<0.001$ vs. DM group, and ${ }^{\# \# / 1} \mathrm{P}<0.001$ vs. Sham group. Data are expressed as the means \pm SD of 3 individual experiments. AIV, astragaloside-IV; DM, diabetes mellitus; RPE cells, retinal pigment epithelial cells. 

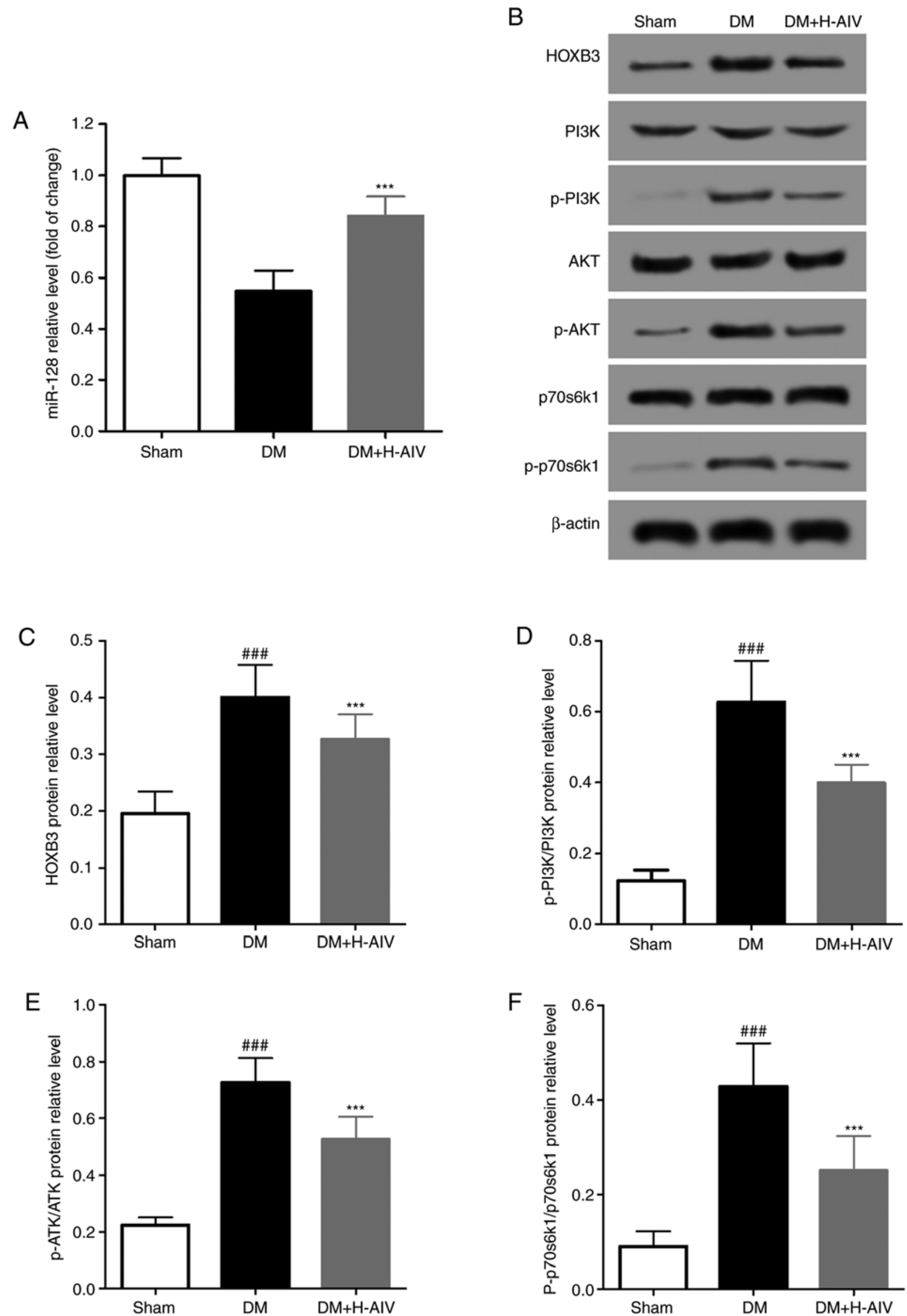

Figure 3. Effect of AIV on the expression of miR-128 in RPE cells from rats with DM. (A) AIV significantly increased miR-128 expression as shown by RT-qPCR; (B) representative protein expression bands in RPE cells from rats with DM; (C-F) statistical analysis of gray value of protein bands; 6 rats in each group. ${ }^{* * *} \mathrm{P}<0.001$ vs. DM group, and ${ }^{\# \# \#} \mathrm{P}<0.001$ vs. Sham group. Data are expressed as the means \pm SD of 3 individual experiments. AIV, astragaloside-IV; DM, diabetes mellitus; RPE cells, retinal pigment epithelial cells. 


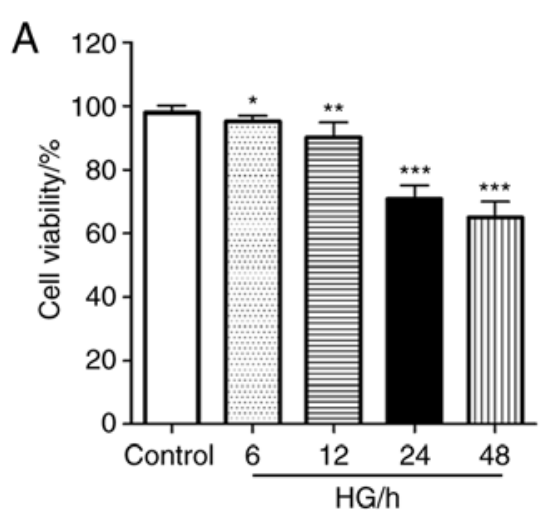

B

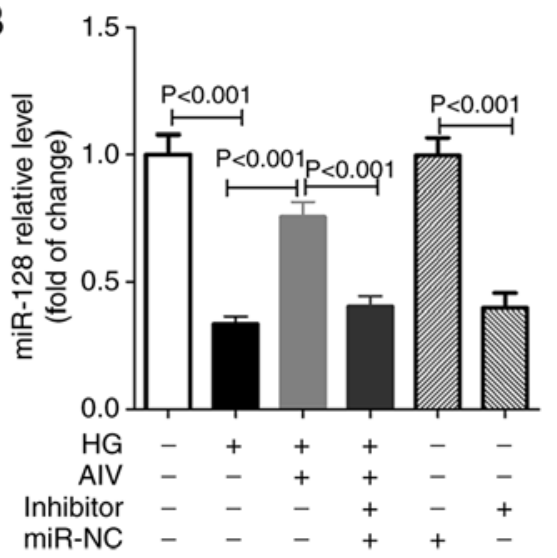

C

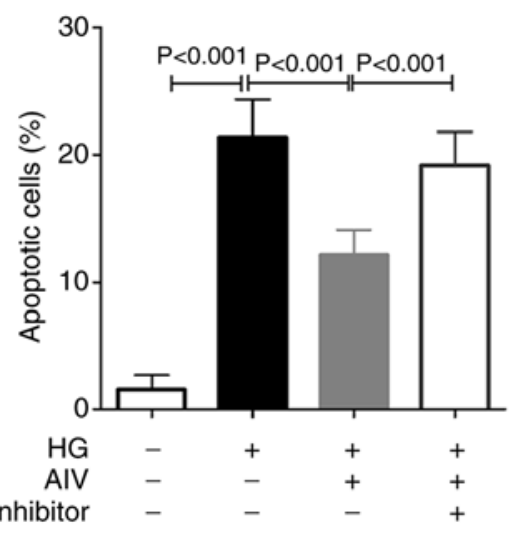

a
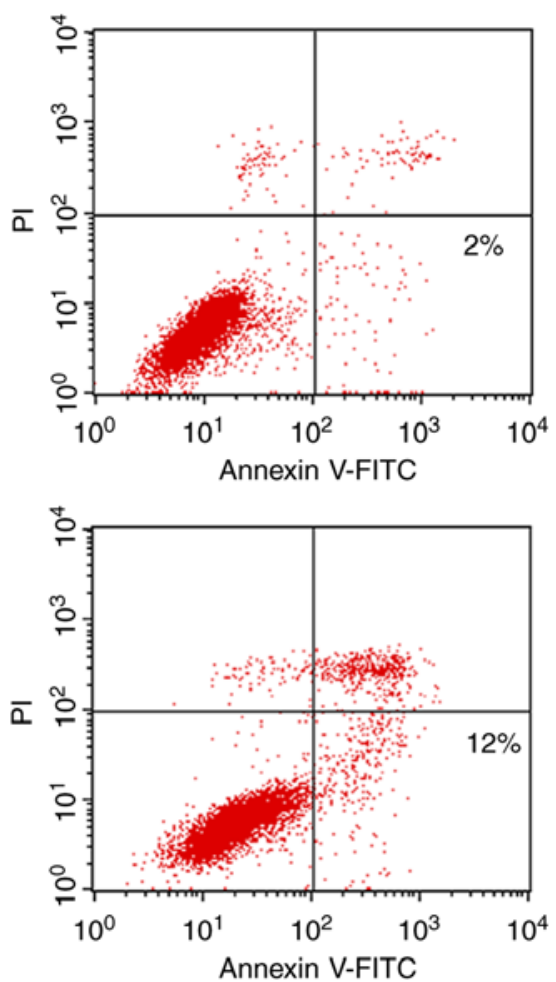

b

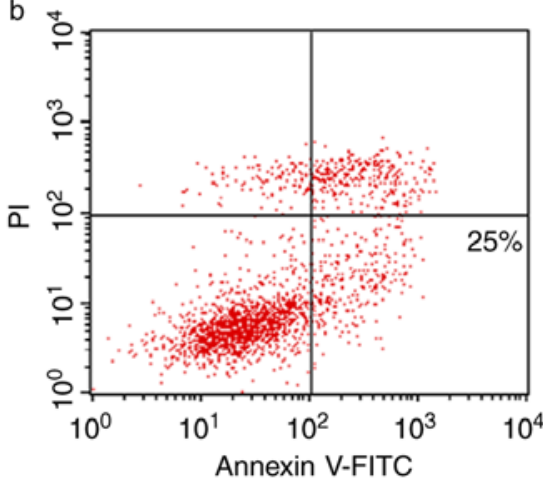

d

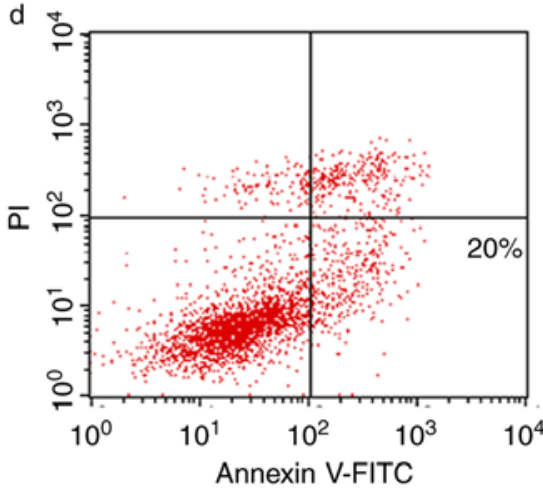

Figure 4. Effect of AIV on apoptosis of rat RPE cells induced by high glucose in vitro. (A) MTT assay was used to assess the viability of rat RPE cells after induction with high glucose at different time points; (B) AIV significantly increased miR-128 expression in rat RPE cells after induction with high glucose at $24 \mathrm{~h}$; (C) AIV protected rat RPE cells after induction with high glucose at $24 \mathrm{~h}$ via miR-128; (D) flow cytometry was used to detect the apoptosis of rat RPE cells following treating with (a) nothing, (b) HG, (c) HG + AIV and (d) HG + AIV + inhibitor; 3 independent experiments per test. Data are expressed as the means \pm SD of 3 individual experiments. ${ }^{*} \mathrm{P}<0.05,{ }^{* *} \mathrm{P}<0.01,{ }^{* * * *} \mathrm{P}<0.001$, compared with control group. AIV, astragaloside-IV; DM, diabetes mellitus; RPE cells, retinal pigment epithelial cells; HG, high glucose; miR-NC, miR-128-inhibitor negative control; inhibitor, miR-128-inhibitor.

was used to detect the level of apoptosis of rat RPE cells. It was found that (Fig. 4C and D) HG treatment induced the apoptosis of RPE cells in vitro, whereas the ratio of apoptotic RPE cells decreased significantly with the administration of AIV treatment. More importantly, compared with the normal RPE cells, the ratio of apoptotic RPE cells increased significantly following the knockdown of miR-128 expression as a result of transfection with miR-128-inhibitor.

As regards the underlying the molecular mechanisms, as shown in Fig. 5, AIV treatment significantly increased the $\mathrm{Bcl}-2, \mathrm{Bcl} 2 / \mathrm{Bax}$ and FasL protein expression levels, which were downregulated by HG in the RPE cells in vitro, and significantly decreased the Bax, Fas, Fas/FasL, active caspase- 3 , active caspase- 8 and active caspase- 9 protein expression levels, which were upregulated by HG in the RPE cells. Moreover, it was found that (Fig. 6) AIV treatment significantly decreased the p-PI3K/PI3K, p-AKT/AKT and p-p70S6K1/p70S6K1 protein expression levels, which were upregulated by HG in RPE cells in vitro. Similarly, compared with the normal RPE cells, the effects of AIV treatment on changes in the expression levles of apoptosis-related proteins and the PI3K/AKT pathway induced by HG were weakened in the RPE cells following miR-128 knockdown.

\section{Discussion}

In the present study, it was first found that AIV not only decreased the blood glucose levels, but also protected the visual 
A

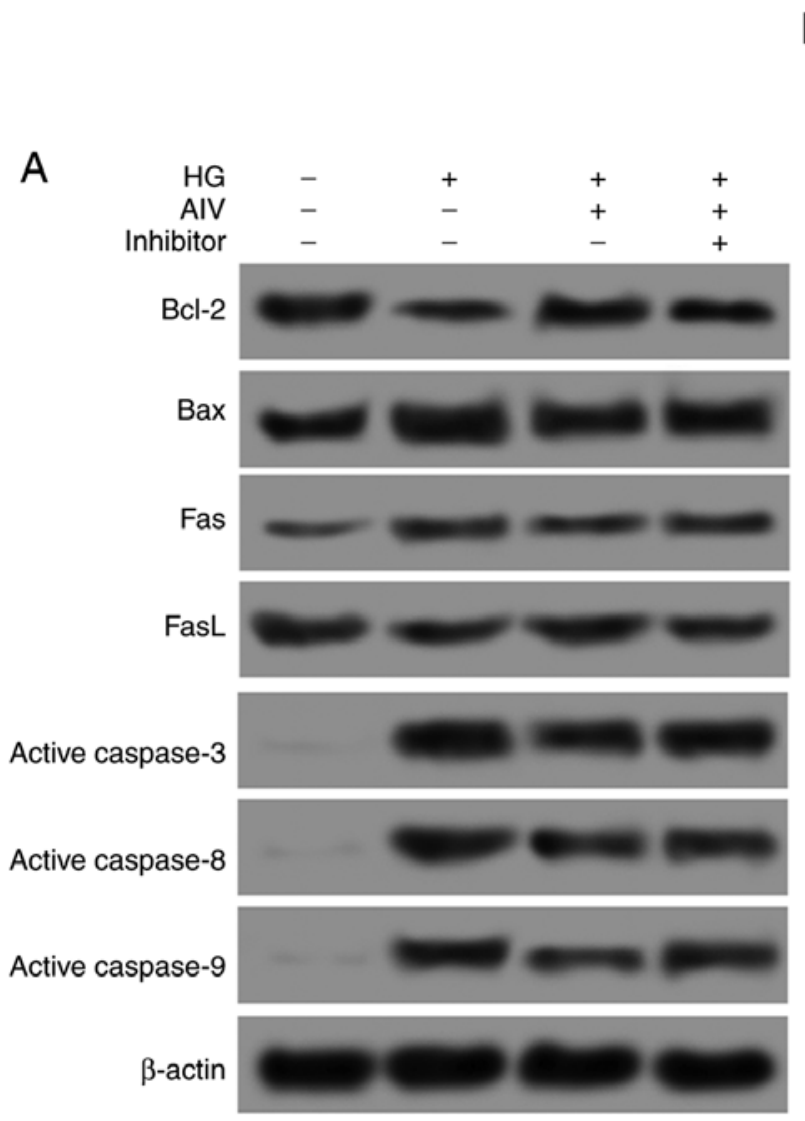

B
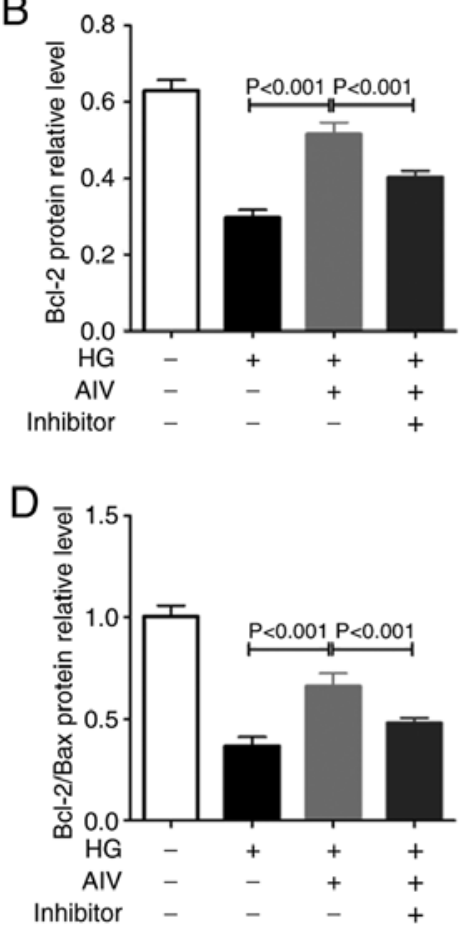

$\mathrm{F}$

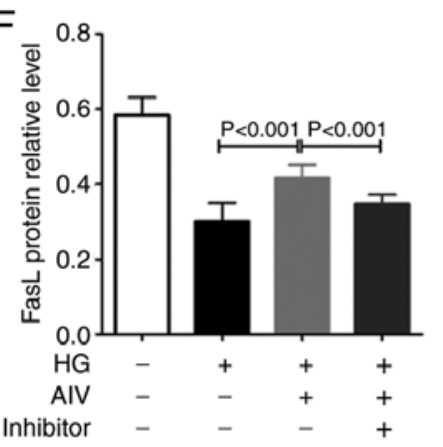

C

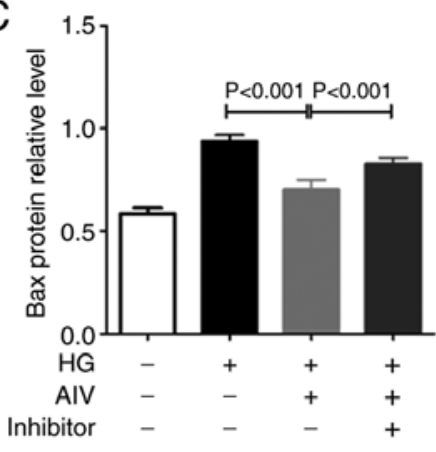

E

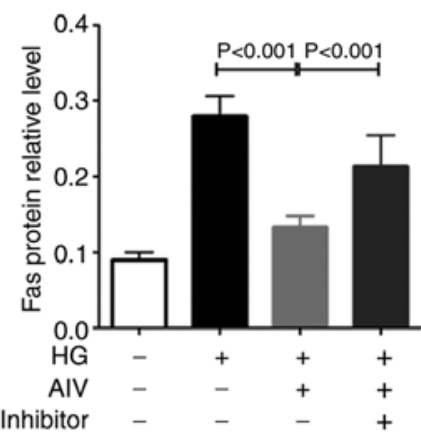

G

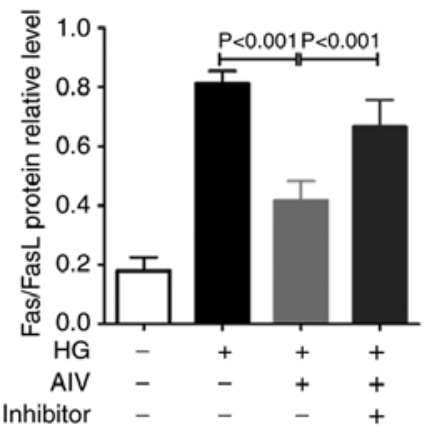

$\mathrm{H}$

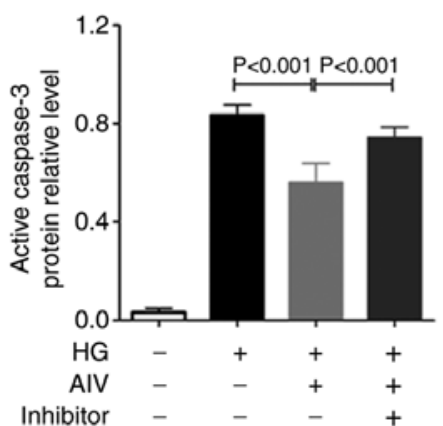

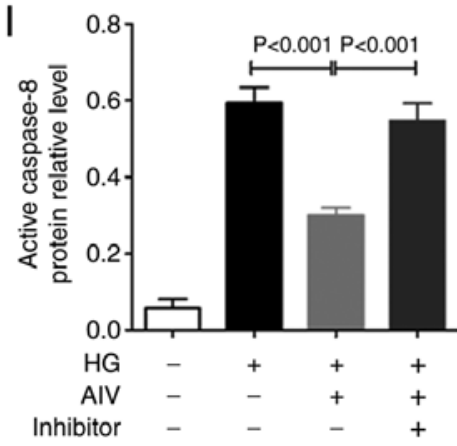

$J$

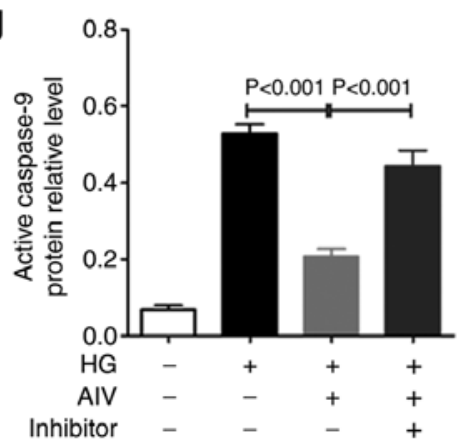

Figure 5. Effect of AIV on apoptosis-related protein expression rat RPE cells in vitro. (A-J) Western blot analysis was used to detect the expression of apoptosis-related protein; 3 independent experiments per test. Data are expressed as the means \pm SD of 3 individual experiments. AIV, astragaloside-IV; DM, diabetes mellitus; RPE cells, retinal pigment epithelial cells; HG, high glucose; miR-NC, miR-128-inhibitor negative control; inhibitor, miR-128-inhibitor.

function of rats with DM. Previous studies have found that AIV plays a role in lowering blood glucose levels in vivo $(16,18)$. Ding et al found that AIV downregulated aldose reductase activity, prevented ERK1/2 phosphorylation and decreased the activity of cytokines, such as nuclear factor $(\mathrm{NF})-\kappa \mathrm{B}$, and significantly decreased retinal ganglion cell apoptosis in $\mathrm{db} / \mathrm{db}$ mice with $\mathrm{DR}$, relieved retinal ganglion cell dysfunction, which proved that AS-IV exerted a certain preventive effect against DR (16). However, the protective effects of AIV against retinopathy have not been extensively studied and the underlying molecular mechanisms remain unclear.

Under normal physiological conditions, RPE cells are essential for maintaining normal retinal functions, such as the absorption of scattered light, retinal pigmentation and synthesis, the renewal and metabolism of photoreceptors, and the formation of blood-retinal barriers. Therefore, they play an irreplaceable role in maintaining visual function. The loss of any type of function of RPE cells can lead to the degradation 

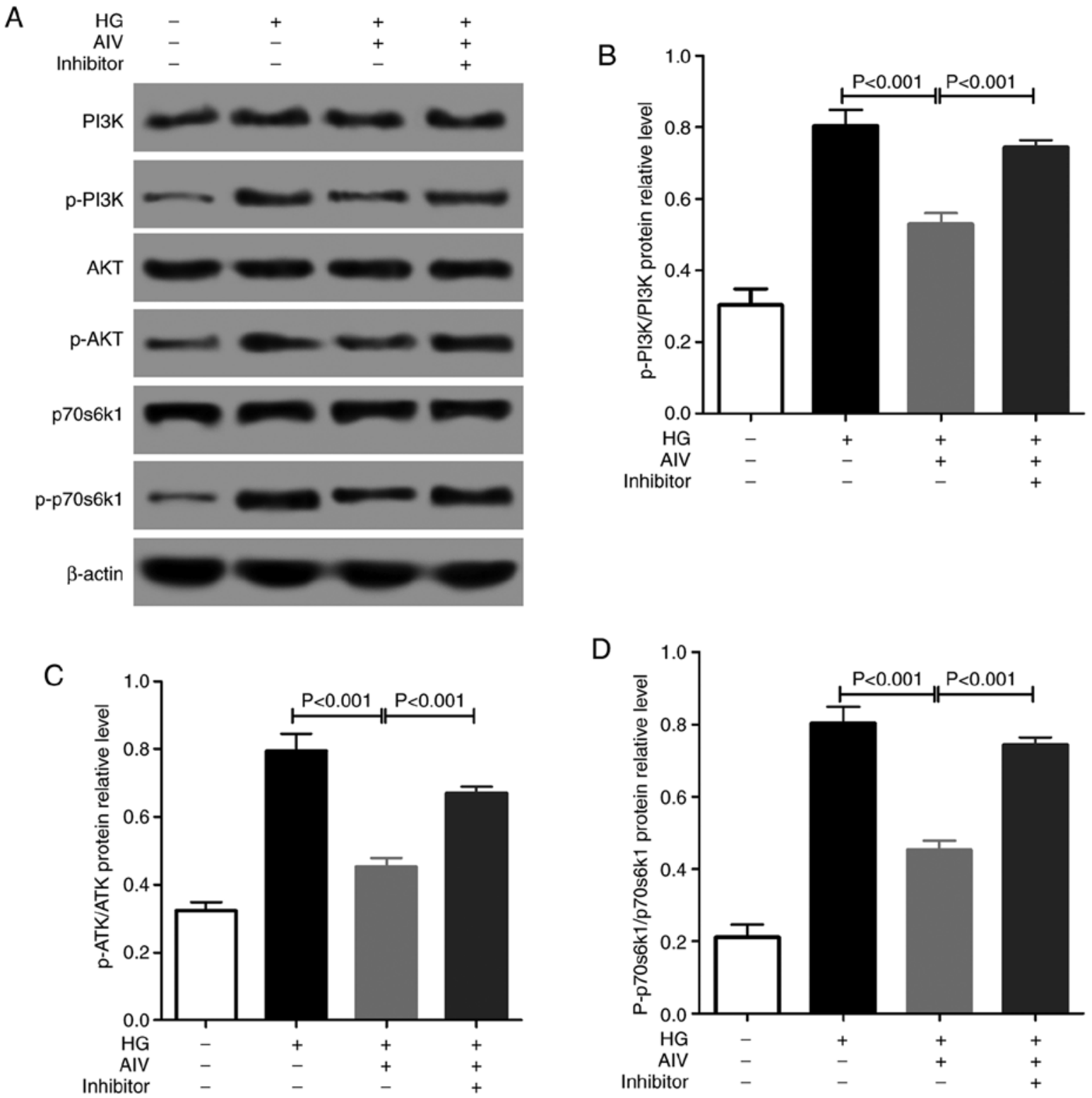

Figure 6. Effect of AIV on PI3K/AKT pathway in rat RPE cells in vitro. (A-D) Western blot analysis was used to detect the expression of p-PI3K, PI3K, p-AKT, AKT, p-p70s6k1 and p70s6k1 protein; 3 independent experiments per test. Data are expressed as the means \pm SD of 3 individual experiments. AIV, astragaloside-IV; DM, diabetes mellitus; RPE cells, retinal pigment epithelial cells; HG, high glucose; miR-NC, miR-128-inhibitor negative control; inhibitor, miR-128-inhibitor.

of the retina, which may result in vision loss and even blindness. Chen et al (19) found that the HG levels induced a decrease in the permeability of ARPE-19 cells in vitro, which induced mitochondrial dysfunction in ARPE-19 cells by decreasing mitochondrial membrane potential and inhibiting Bcl-2 protein levels, which induced apoptosis by promoting the S0CS1 and Fas/FasL signaling pathways. Crider et al (20) found that HG levels affected water transport from the retina to the choroid by inducing a loss of $\mathrm{Na}^{+}-\mathrm{K}^{+}$-ATPase function in bovine RPE cells. In the present study, it ws found that AIV significantly attenuated the apoptosis of REP cells from rats with DM. This indicated that the protective effects of AIV on retinopathy in rats with DM may be related to the inhibition of RPE cell apoptosis.

Although to the best of our knowledge, no previous studies to date have demonstrated that AIV can protect RPE cells in rats with DM, previous studies have found that
AIV can inhibit inflammatory and oxidative stress-induced apoptosis. Yin et al (21) found that AIV attenuated myocardial ischemia/reperfusion injury in rats via the inhibition of calcium-sensing receptor-mediated apoptotic signaling pathways, while Xiong et al (22) found that AIV inhibited guinea pig cochlear impulse noise-induced apoptosis by inhibiting reactive oxygen species (ROS) production. Apoptosis is a complex process involving a number of different mechanisms, such as the mitochondrial pathway, the Fas death receptor pathway and the endoplasmic reticulum stress pathway (23). In the mitochondrial programmed death pathway, cytochrome $c$ released from the mitochondria binds to the Apaf-1 protein in the cytoplasm to increase the affinity of Apaf- 1 to dATP/ATP, and then dATP/ATP binds to the cytC/Apaf-1 complex to promote the oligomerization of the cytC/Apaf-1 complex, thereby forming apoptosomes, which are able to cause the auto-activation of caspase- 3 , caspase- 8 and caspase- 9 , 
resulting in chromosomal condensation, nuclear DNA fragmentation and nuclear membrane rupture, which ultimately lead to cell death $(24,25)$. In the present study, it was found that AIV significantly decreased the protein expression levels of $\mathrm{Bax} / \mathrm{Bcl} 2$, active caspase-3, active caspase- 8 and active caspase-9 in RPE cells from rats with DM. Furthermore, it was found that AIV significantly decreased the protein expression of Fas, and significantly increased the expression of FasL and Fas/FasL. In the Fas death receptor pathway, apoptotic signals induce the increased expression of Fas, and apoptotic bodies are formed by FasL to induce apoptosis $(26,27)$. Therefore, these results suggested that the apoptosis of RPE cells from rats with DM was regulated by multiple pathways, including at least the mitochondrial apoptosis pathway and the Fas death receptor pathway.

A previous study by the authors found that miR-128 attenuated the apoptosis induced by HG in RPE cells in vitro (12). It was also found that miR-128 expression was downregulated, whereas AIV treatment significantly increased the expression of miR-128 and decreased the expression levels of p-PI3K/PI3K, p-AKT/AKT and p-p70S6K1/p70S6K1 in RPE cells from rats with DM. This suggests that miR-128 may also protect RPE cells in a HG environment in vivo and AIV treatment may protect RPE cells by increasing the expression of miR-128. The PI3K/Akt pathway is an important signaling pathway that regulates cell survival and apoptosis (28), and has been found to be closely related to mitochondria-mediated apoptosis pathway (29) and the Fas death receptor pathway (19). In the PI3K/Akt pathway, phosphatidylinositol-dependent kinase 1 (PDK1) binds to Akt to phosphorylate Akt, which in turn activates p70S6K1, which ultimately results in the inhibition of cell apoptosis $(30,31)$. Therefore, AIV may be able to protect RPE cells by increasing miR-128 expression via the $\mathrm{PI} 3 \mathrm{~K} / \mathrm{AKT}$ pathway in rats with DM.

In order to investigate whether miR-128 was the target of AIV in RPE cells of DM rats, RPE cells were isolated from healthy rats and miR-128 expression was knocked down by transfection with an miR-128-inhibitor into RPE cells. It was found that compared with normal RPE cells, the ratio of HG-induced apoptotic RPE cells was significantly higher following the knockdown of miR-128 expression by transfection with a miR-128-inhibitor.

A limitation of the present study was that since no miR-128 or PI3K/AKT knockout rats were used, the results obtained from in vitro experiments need to be validated in vivo.

In conclusion, the present study demonstrates that treatment of rats with $60 \mathrm{mg} / \mathrm{kg}$ AIV may be able to inhibit the apoptosis of RPE cells by upregulating the expression of miR-128 in DM rats, and its specific molecular mechanisms may be related to the PI3K/AKT-mediated mitochondrial apoptosis pathway and the Fas death receptor pathway. Therefore, our study identified $60 \mathrm{mg} / \mathrm{kg}$ AIV as a potential drug for the prevention and treatment of diabetic retinopathy. However, the limitations of the present study should be noted and further studies on miR-128 knockout in rats may aid in the identification of the specific molecular mechanism of action of AIV.

\section{Acknowledgements}

Not applicable.

\section{Funding}

No funding was received.

\section{Availability of data and materials}

All data generated or analyzed during this study are included in this published article.

\section{Authors' contributions}

CS was involved in the conception and design of the study. TW and $\mathrm{ZZ}$ analyzed the experimental data. LS, XS, QQ and JL performed the experiments. TW, ZZ and CS were the major contributors in writing the manuscript. All authors read and approved the final manuscript.

\section{Ethics approval and consent to participate}

The animal experiments performed in the present study were approved and supervised by the Animal Care and Use Committee of Weihai Municipal Hospital, and conformed with guidelines of the National Institution of Health.

\section{Patient consent for publication}

Not applicable.

\section{Competing interests}

The authors declare that they have no competing interests.

\section{References}

1. Frank RN: Diabetic retinopathy. N Engl J Med 350: 48-58, 2004.

2. Roglic G: WHO Global report on diabetes: A summary. Int $\mathbf{J}$ Noncommun Dis 1: 3-8, 2016.

3. Zheng Y, He M and Congdon N: The worldwide epidemic of diabetic retinopathy. Indian J Ophthalmol 60: 428-431, 2012.

4. Simó R, Villarroel M, Corraliza L, Hernández C and Garcia-Ramírez M: The retinal pigment epithelium: Something more than a constituent of the blood-retinal barrier-implications for the pathogenesis of diabetic retinopathy. J Biomed Biotechnol 2010: 190724, 2010.

5. Chiba C: The retinal pigment epithelium: An important player of retinal disorders and regeneration. Exp Eye Res 123: 107-114, 2014.

6. Spekker-Bosker K, Ufermann CM, Oldenburg M, Däubener W and Eller SK: Interplay between IDO1 and iNOS in human retinal pigment epithelial cells. Med Microbiol Immunol 208: 811-824, 2019.

7. Tsao YP, Ho TC, Chen SL and Cheng HC: Pigment epithelium-derived factor inhibits oxidative stress-induced cell death by activation of extracellular signal-regulated kinases in cultured retinal pigment epithelial cells. Life Sci 79: 545-550, 2006.

8. Seagle BL, Gasyna EM, Mieler WF and Norris JR Jr: Photoprotection of human retinal pigment epithelium cells against blue light-induced apoptosis by melanin free radicals from Sepia officinalis. Proc Natl Acad Sci USA 103: 16644-16648, 2006.

9. Steindl K and Binder S: Retinal degeneration processes and transplantation of retinal pigment epithelial cells: Past, present and future trends. Spektrum der Augenheilkunde 22: 357-361, 2008.

10. Ren S, Zhang H, Mu Y, Sun M and Liu P: Pharmacological effects of Astragaloside IV: A literature review. J Tradit Chin Med 33: 413-416, 2013.

11. Lia L, Houb X, Xub R, Liua C and Tub M: Research review on the pharmacological effects of astragaloside IV. Fundam Clin Pharmacol 31: 17-36, 2017. 
12. Zhang Z, Sun J, Hu Y, Song $\mathrm{C}$ and $\mathrm{Wu} X$ : miR-128 protects retinal pigment epithelium in high glucose through HOXB3/PI3K/ ERK-mTOR pathway. Int J Clin Exp Med 9: 1684-1691, 2016.

13. Chakraborty $\mathrm{C}$, George Priya Doss $\mathrm{C}$ and Bandyopadhyay S: miRNAs in insulin resistance and diabetes-associated pancreatic cancer: The 'minute and miracle' molecule moving as a monitor in the 'genomic galaxy'. Curr Drug Targets 14: 1110-1117, 2013.

14. Chakraborty C,Doss CG,Bandyopadhyay S and Agoramoorthy G: Influence of miRNA in insulin signaling pathway and insulin resistance: Micro-molecules with a major role in type- 2 diabetes. Wiley Interdiscip Rev RNA 5: 697-712, 2014.

15. Ciccacci C, Morganti R, Di Fusco D, D'Amato C, Cacciotti L, Greco C, Rufini S, Novelli G, Sangiuolo F, Marfia GA, et al: Common polymorphisms in MIR146a, MIR128a and MIR27a genes contribute to neuropathy susceptibility in type 2 diabetes. Acta Diabetol 51: 663-671, 2014.

16. Ding Y, Yuan S, Liu X, Mao P, Zhao C, Huang Q, Zhang R, Fang Y, Song Q, Yuan D, et al: Protective effects of astragaloside IV on $\mathrm{db} / \mathrm{db}$ mice with diabetic retinopathy. PLoS One 9: e112207, 2014

17. Livak KJ and Schmittgen TD: Analysis of relative gene expression data using real-time quantitative PCR and the 2(-Delta Delta $\mathrm{C}(\mathrm{T})$ ) method. Methods 25: 402-408, 2001.

18. Gui D, Huang J, Guo Y, Chen J, Chen Y, Xiao W, Liu X and Wang N: Astragaloside IV ameliorates renal injury in streptozotocin-induced diabetic rats through inhibiting NF- $\mathrm{kB}$-mediated inflammatory genes expression. Cytokine 61: 970-977, 2013.

19. Chen M, Wang W, Ma J, Ye P and Wang K: High glucose induces mitochondrial dysfunction and apoptosis in human retinal pigment epithelium cells via promoting SOCS1 and Fas/FasL signaling. Cytokine 78: 94-102, 2016.

20. Crider JY, Yorio T, Sharif NA and Griffin BW: The effects of elevated glucose on $\mathrm{Na}+/ \mathrm{K}(+)$-ATPase of cultured bovine retinal pigment epithelial cells measured by a new nonradioactive rubidium uptake assay. J Ocul Pharmacol Ther 13: 337-352, 1997.

21. Yin B, Hou XW and Lu ML: Astragaloside IV attenuates myocardial ischemia/reperfusion injury in rats via inhibition of calcium-sensing receptor-mediated apoptotic signaling pathways. Acta Pharmacol Sin 40: 599-607, 2019.
22. Xiong $\mathrm{M}, \mathrm{He} \mathrm{Q}$, Lai $\mathrm{H}$ and Wang J: Astragaloside IV inhibits apoptotic cell death in the guinea pig cochlea exposed to impulse noise. Acta Otolaryngol 132: 467-474, 2012.

23. Hengartner MO: The biochemistry of apoptosis. Nature 407: 770-776, 2000

24. Ugarte-Uribe B and García-Sáez AJ: Apoptotic foci at mitochondria: In and around Bax pores. Philos Trans R Soc Lond B Biol Sci 372: pii: 20160217, 2017.

25. Olson M and Kornbluth S: Mitochondria in apoptosis and human disease. Curr Mol Med 1: 91-122, 2001.

26. Stel AJ, Ten Cate B, Jacobs S, Kok JW, Spierings DC, Dondorff M, Helfrich W, Kluin-Nelemans HC, de Leij LF, Withoff S and Kroesen BJ: Fas receptor clustering and involvement of the death receptor pathway in rituximab-mediated apoptosis with concomitant sensitization of lymphoma B cells to fas-induced apoptosis. J Immunol 178: 2287-2295, 2007.

27. Nagata S and Golstein P: The Fas death factor. Science 267: 1449-1456, 1995

28. Franke TF, Hornik CP, Segev L, Shostak GA and Sugimoto C: PI3K/Akt and apoptosis: Size matters. Oncogene 22: 8983-8998, 2003.

29. Tsuruta F, Masuyama N and Gotoh Y: The phosphatidylinositol 3-kinase (PI3K)-Akt pathway suppresses Bax translocation to mitochondria. J Biol Chem 277: 14040-14047, 2002.

30. Zhao P, Meng Q, Liu LZ, You YP, Liu N and Jiang BH: Regulation of survivin by PI3K/Akt/p70S6K1 pathway. Biochem Biophys Res Commun 395: 219-224, 2010.

31. Chai X, Sun D, Han Q, Yi L, Wu Y and Liu X: Hypoxia induces pulmonary arterial fibroblast proliferation, migration, differentiation and vascular remodeling via the PI3K/Akt/p70S6K signaling pathway. Int J Mol Med 41: 2461-2472, 2018.

This work is licensed under a Creative Commons Attribution-NonCommercial-NoDerivatives 4.0 International (CC BY-NC-ND 4.0) License. 